Pacific Journal of Mathematics

ISOMORPHISMS BETWEEN HARMONIC AND $P$-HARMONIC 


\section{ISOMORPHISMS BETWEEN HARMONIC AND $P$-HARMONIC HARDY SPACES ON RIEMANN SURFACES}

\section{J. L. SCHIFF}

In this paper we investigate the relationship between the Hardy space $H^{q}(R)$ of harmonic functions on a hyperbolic Riemann surface $R$, and the Hardy space $P^{q}(R)$ of solutions of the equation $\Delta u=P u$, where $P \geqq 0, P \not \equiv 0$ is a $C^{1}$-density on $R$. Under certain conditions these spaces are shown to be canonically isomorphic, although in general this is not the case. However, specific subspaces are found which are isomorphic and their relationship with other function spaces is discussed.

1. Introduction. Hardy spaces on Riemann surfaces have been studied by Heins [2], Schiff [11], in the setting of $\Phi$-bounded functions by Parreau [8], and in the general context of harmonic spaces by Lumer-Naim [4], among others. The present work, in the setting of a hyperbolic Riemann surface $R$, examines the Hardy space $P^{q}(R)$ for the equation $\Delta u=P u, P \geqq 0, P \neq 0$, which thus falls within the framework of [4]. Hence, the results contained therein will be applicable to our study of the isomorphic relations between the harmonic Hardy space $H^{q}(R)$ and $P^{q}(R)$. In general, no such isomorphic relation between $H^{q}(R)$ and $P^{q}(R)$ exists, yet when particular subspaces are considered, an isomorphism is shown to indeed exist between the specified subspaces.

In the fourth section we investigate the conditions under which the inclusion relations are strict or not, between the various spaces which have been introduced. Certain isomorphisms are obtained under new conditions in the final section.

Some of the results obtained have natural generalizations to harmonic spaces and to $\Phi$-bounded $P$-harmonic functions for a convex increasing $\Phi$, however, these aspects of the theory will not be treated here.

2. Preliminaries. Let $R$ be a hyperbolic Riemann surface and $P \geqq 0, P \neq \equiv$ a $C^{1}$-density on $R$. We denote by $P B(R)$ (resp. $H B(R))$ the space of bounded $C^{2}$-solutions on $R$ of the elliptic equation $\Delta u=P u(\Delta u=0)$, and by $P B^{\prime}(R)$ (resp. $H B^{\prime}(R)$ ) the quasibounded counterpart. A $C^{2}$-solution of $\Delta u=P u$ is called a $P$-harmonic function, and the space of such functions on an open set $U$ of $R$ is denoted by $P(U) . \quad H(U)$ denotes the space of harmonic functions on $U$. A 
$C^{2}$-function $s$ is $P$-superharmonic if and only if $\Delta s \leqq P s$. If $s$ is $P$-superharmonic, $-s$ is $P$-subharmonic. Refer to Royden [9] for details concerning $P$-harmonic functions.

Associated with $R$ is the Wiener compactification $R^{*}$ of $R$, the Wiener ideal boundary $\Gamma=R^{*}-R$, and the Wiener harmonic boundary $\Delta \subset \Gamma$. We will also make use of the following maximum principle for the class $H B^{\prime}(R)$, the functions of which have continuous extensions to $\Delta$.

Proposition 1. Let $u \in H B^{\prime}(R)$ with

$$
m \leqq u \leqq M
$$

on $\Delta$. Then $m \leqq u \leqq M$ on $R$.

The reader is referred to Chapter IV of Sario-Nakai [10] for a comprehensive treatment of the Wiener compactification and the $H B^{\prime}$ maximum principle.

By a regular exhaustion $\{\Omega\}$ of $R$ we mean an exhaustion of $R$ by relatively compact open subsets $\Omega$ of $R$ such that $\partial \Omega$ is analytic.

We proceed to define the Hardy space for $\Delta u=P u$. Let $\{\Omega\}$ be a regular exhaustion of $R$ and $z_{0} \in R$ a fixed point. Denote by $\mu_{z_{0}}^{\Omega}$ the $P$-harmonic measure on $\partial \Omega$ relative to $z_{0}$ and $\Omega$. Clearly $\int_{\partial \Omega} d \mu_{z_{0}}^{\Omega} \leqq 1$ for all $\Omega$.

Definition. A function $u \in P^{q}(R), 1 \leqq q<\infty$, if for some constant $M \geqq 0$,

$$
\|u\|_{q, \Omega}=\left(\int_{\partial \Omega}|u|^{q} d \mu_{z_{0}}^{\Omega}\right)^{1 / q} \leqq M
$$

for all $\Omega$ of a regular exhaustion $\{\Omega\}$ of $R$.

That the definition of $P^{q}(R)$ is independent of the choice of $z_{0}$ or the particular exhaustion, is a consequence of the following (cf. Lumer-Naim [4]):

Proposition 2. $u \in P^{q}(R), 1 \leqq q<\infty$, if and only if $|u|^{q}$ has a $P$-harmonic majorant.

A further result of [4] is quoted here as it will be useful in the sequel.

Proposition 3. Every $u \in P^{q}(R)$ (resp. $H^{q}(R)$ ) is the difference of two positive $P$-harmonic (resp. harmonic) functions in $P^{q}(R)$ (resp. $\left.H^{q}(R)\right), 1 \leqq q<\infty$, and conversely. 
3. Isomorphisms. Let $\Omega$ be a regular subregion of $R$ and $\phi$ a continuous function on $\partial \Omega$. We denote by $P_{\phi}^{\Omega}$ the function belonging to $P(\Omega) \cap C(\bar{\Omega})$ such that $P_{\phi}^{\Omega} \mid \partial \Omega=\phi$. The harmonic counterpart to $P_{\phi}^{\Omega}$ is denoted by $H_{\phi}^{\Omega}$.

In this section we assume that $1 \leqq q<\infty$.

Define a linear operator $\lambda_{P}: H^{q}(R) \rightarrow P^{q}(R)$ by

$$
\lambda_{P} h=\lim _{\Omega \rightarrow R} P_{h}^{\Omega}
$$

To show that $\lambda_{P}$ is well-defined, let $h \in H^{q}(R)$. Then $h=h_{1}-h_{2}$, $h_{t} \in H^{q}(R), \quad h_{\imath} \geqq 0, \quad i=1,2$, by Proposition 3 . By the $P$-harmonic maximum principle (cf. Royden [9]), $\left\{P_{h_{l}}^{\Omega}\right\}$ is decreasing and

$$
0 \leqq P_{h_{t}}^{\Omega} \leqq h_{\imath}
$$

on $\Omega$. Letting $\Omega \rightarrow R, P_{h_{t}}^{\Omega}$ converges to a function $P_{h_{t}} \in P(R)$, and therefore

$$
P_{h}^{\Omega}=P_{h_{1}}^{\Omega}-P_{h_{2}}^{\Omega} \rightarrow P_{h_{1}}-P_{h_{2}}=\lambda_{P} h \in P(R) .
$$

Moreover,

$$
\left|\lambda_{P} \boldsymbol{h}\right|^{q} \leqq 2^{q}\left(\left|P_{h_{1}}\right|^{q}+\left|P_{h_{2}}\right|^{q}\right) \leqq 2^{q}\left(h_{1}^{q}+h_{2}^{q}\right) \leqq h^{\prime}
$$

for some $h^{\prime} \in H(R)$. Since the $P$-subharmonic function $\left|\lambda_{P} h\right|^{q}$ has the $P$-superharmonic majorant, $h^{\prime}$, a standard Perron family argument shows that $\left|\lambda_{P} h\right|^{q}$ has a $P$-harmonic majorant, implying $\lambda_{P} h \in P^{q}(R)$.

Note that for $h \in H^{q}(R), h>0, \lambda_{P} h$ is the greatest $P$-harmonic minorant of $h$, and consequently $h-\lambda_{P} h$ is a $P$-potential, i.e. a positive $P$-superharmonic function whose greatest $P$-harmonic minorant is zero.

Next, let

$$
\Lambda_{P}^{q}(R)=\left\{u \in P(R):|u|^{q} \leqq h \text { for some } h \in H(R)\right\} .
$$

The inequality $|t|^{p} \leqq 1+|t|^{q}$ for $1 \leqq p \leqq q<\infty$ implies:

Proposition 4. $\quad \Lambda_{P}^{q}(R) \subset \Lambda_{P}^{p}(R) \subset \Lambda_{P}^{1}(R)$.

The previous Perron family considerations also yield:

Proposition 5. $P B(R) \subset \Lambda_{P}^{q}(R) \subset P^{q}(R)$.

We further establish: 
Proposition 6. If $u \in \Lambda_{P}^{q}(R)$, then $u=u_{1}-u_{2}, 0 \leqq u_{i} \in \Lambda_{P}^{q}(R), i=$ 1,2 .

Proof. Since $u \in \Lambda_{P}^{1}(R)$ by Proposition $4,|u|$ has a $P$-harmonic majorant. Denoting by $U^{+}, U^{-}$, the least $P$-harmonic majorant of $u^{+}=u \cup 0$, and $u^{-}=(-u) \cup 0$ resp., it follows that $u=U^{+}-U^{-}$. Moreover, $u \in \Lambda_{P}^{q}(R)$ implies $|u|^{q} \leqq h \in H(R)$ and therefore $\left(u^{+}\right)^{q} \leqq h$.

Consequently

$$
\begin{aligned}
\left(U^{+}\right)^{q}\left(z_{0}\right) & =\lim _{\Omega \rightarrow R}\left(\int_{\partial \Omega} u^{+} d \mu_{z_{0}}^{\Omega}\right)^{q} \leqq \lim _{\Omega \rightarrow R} \int_{\partial \Omega}\left(u^{+}\right)^{q} d \mu_{z_{0}}^{\Omega} \\
& \leqq \lim _{\Omega \rightarrow R} \int_{\partial \Omega} h d \mu_{z 0}^{\Omega} \leqq h\left(z_{0}\right),
\end{aligned}
$$

where the last inequality follows from the fact that $h$ is $P$ superharmonic. Hence $U^{+} \in \Lambda_{P}^{q}(R)$. Similarly $U^{-} \in \Lambda_{P}^{q}(R)$ and thus $u \in \Lambda_{P}^{q}(R)$, as desired.

The space $\Lambda_{P}^{q}(R)$ is related to $H^{q}(R)$ in the following way.

THEOREM 1. $\lambda_{P}\left(H^{q}(R)\right)=\Lambda_{P}^{q}(R)$.

Proof. It suffices to show $\lambda_{P}\left(H^{q}(R)\right) \supset \Lambda_{P}^{q}(R)$ since the inclusion $\lambda_{P}\left(H^{q}(R)\right) \subset \Lambda_{P}^{q}(R)$ is obvious from the definition of $\lambda_{P}$.

Let $0 \leqq u \in \Lambda_{P}^{q}(R)$. Then $u^{q} \leqq h$ for some $h \in H(R)$, and since $u \in \Lambda_{P}^{1}(R), u$ has a harmonic majorant. Let $h_{u}$ be the least harmonic majorant of $u$. Thus for a regular exhaustion $\{\Omega\}$ of $R$,

$$
h_{u}=\lim _{\Omega \rightarrow R} h_{u}^{\Omega}
$$

where $h_{u}^{\Omega}|\partial \Omega=u| \partial \Omega, h_{u}^{\Omega} \in H(\Omega) \cap C(\bar{\Omega})$. Therefore

$$
\left(h_{u}^{\Omega}\right)^{q}\left|\partial \Omega=u^{q}\right| \partial \Omega \leqq h,
$$

implying $\left(h_{u}^{\Omega}\right)^{q} \leqq h$ on $\Omega$. Letting $\Omega \rightarrow R$ yields

$$
h_{u}^{q} \leqq h
$$

on $R$, and $h_{u} \in H^{q}(R)$.

In view of the fact that $h_{u}-u$ is a $P$-potential, $u$ is the greatest $P$-harmonic minorant of $h_{u}$, i.e. $\lambda_{P} h_{u}=u$.

For an arbitrary $u \in \Lambda_{p}^{q}(R), u=u_{1}-u_{2}, 0 \leqq u_{i} \in \Lambda_{P}^{q}(R), i=1,2$, and we have $h_{u}=h_{u_{1}}-h_{u_{2}}$, which proves the theorem. 
We construct a new operator which will turn out to be the inverse of $\lambda_{P}$ in certain instances.

Let $u \in P^{q}(R), u=u_{1}-u_{2}, 0 \leqq u_{1} \in P^{q}(R), i=1,2$, and consider the increasing family of harmonic functions $\left\{H_{u_{1}}^{\Omega}\right\}$. Set

$$
H_{u_{t}}=\lim _{\Omega \rightarrow R} H_{u_{i}}^{\Omega}
$$

whenever the limit exists. In this case

$$
H_{u}^{\Omega}=H_{u_{1}}^{\Omega}-H_{u_{2}}^{\Omega} \rightarrow H_{u_{1}}-H_{u_{2}} \in H(R),
$$

and we define

$$
\mu_{p} u=\lim _{\Omega \rightarrow R} H_{u}^{\Omega} .
$$

Observe that for $u>0, \mu_{P} u$, when it is defined, is the least harmonic majorant of $u$ on $R$.

Proposition 7. $\Lambda_{P}^{q}(R) \subset$ domain $\left(\mu_{P}\right)$ and $\mu_{P}: \Lambda_{P}^{q}(R) \rightarrow H^{q}(R)$.

The proof is analogous to that of Theorem 1.

It is clear that $\mu_{P}$ is linear on $\Lambda_{P}^{q}(R)$. Set $M_{P}^{q}(R)=\mu_{P}\left(\Lambda_{p}^{q}(R)\right)$.

THEOREM 2. $\mu_{P}: \Lambda_{P}^{q}(R) \rightarrow M_{P}^{q}(R)$ is an isomorphism with inverse $\lambda_{P}: M_{P}^{q}(R) \rightarrow \Lambda_{P}^{q}(R)$.

Proof. Let $u \in \Lambda_{P}^{q}(R)$. Then $u=u_{1}-u_{2}, \quad 0 \leqq u_{\imath} \in \Lambda_{P}^{q}(R), \quad i=$ 1,2. It follows that $\mu_{p} u_{\imath} \in M_{P}^{q}(R)$ and $\mu_{P} u_{\imath}=u_{\imath}+p_{t}$, where $p_{\imath}$ is a $P$-potential. Therefore

$$
\left(\lambda_{P} \circ \mu_{P}\right)\left(u_{\imath}\right)=\lambda_{P}\left(u_{\imath}+p_{\imath}\right)=u_{t}
$$

$i=1,2$, and hence $\lambda_{P} \circ \mu_{P}=$ identity.

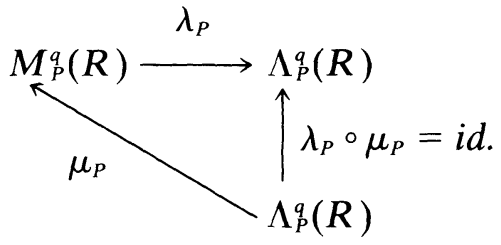


We deduce that $\mu_{P}$ is injective, hence an isomorphism, and that $\lambda_{P}$ is surjective. Moreover, it is easily seen that $\lambda_{P}$ is injective and $\mu_{P} \circ \lambda_{P}=$ identity.

4. Examples. In this section we give some examples demonstrating the proper inclusion relations between various function spaces that have been considered.

THEOREM 3. There is a Riemann surface $R$ and a density $P$ for which $M_{P}^{q}(R) \varsubsetneqq H^{q}(R), 1 \leqq q<\infty$.

Proof. Take a Riemann surface $R \in \mathscr{O}_{P B}-\mathscr{O}_{G}$, that is $R$ is hyperbolic, yet $P B(R)=\{0\}$ (cf. e.g. Royden [9] for the existence of such a surface). Then for any positive constant $c$, we have $c \in H^{q}(R)$. However, there is no greatest $P$-harmonic minorant of $c$ other than zero, and hence $\lambda_{P} c=0$.

Since $\lambda_{P}$ is injective on $M_{P}^{q}(R)$ by Theorem 2 , and $\lambda_{P} 0=0$, we deduce that $c \in H^{q}(R)-M_{P}^{q}(R)$.

The $P$-harmonic Hardy class $P^{a}(R)$ is related to other function spaces by the following string of inclusions (cf. Lumer-Naim [4]):

Proposition 8. $P B(R) \subset \Lambda_{p}^{q}(R) \subset P^{q}(R) \subset P B^{\prime}(R)$, for $1<q<\infty$.

By the way of contrast with the harmonic case, observe that in the preceding example, $M_{P}^{q}(R)=\{0\}$, and thus $H B(R) \not \subset M_{P}^{q}(R)$.

Using a similar proof as for that given in the harmonic case (Schiff [11]), it can be shown that:

Theorem 4. If $\operatorname{dim} P B(R)=n<\infty$, then

$$
P B(R)=\Lambda_{P}^{q}(R)=P^{q}(R)=P B^{\prime}(R),
$$

for $1<q<\infty$.

In general this may not be the case. Some further preliminaries at this juncture are in order.

It has been demonstrated (cf. eg. Nakai [7], also Royden [9]), that the Banach spaces $P B(R)$ and $H B(R)$ are canonically isomorphic, that is, there exists a bijective linear isometry $T: P B(R) \rightarrow H B(R)$ such that for $u \in P B(R),|u-T u|$ is dominated by a potential, whenever the following condition is valid:

$$
\int_{R-K} G(z, \zeta) P(\zeta) d \xi d \eta<\infty \quad(\zeta=\xi+i \eta)
$$


Here $K$ is a $B$-negligible subset of $R$, that is $R^{*}-\bar{K}$ is a neighborhood of $\Delta$ (cf. Nakai [7]), $G(z, \zeta)$ is the harmonic Green's function on $R$, and (*) is assumed to be valid for one (hence for all) $z \in R$.

In view of Theorem 4 and the preceding remarks, we conclude:

Proposition 9. If $\operatorname{dim} H B=n<\infty$, and condition (*) is valid, then $H^{q}(R)$ is canonically isomorphic to $P^{q}(R), 1<q<\infty$.

Our next two theorems (nos. 5 and 6) require a result from ideal boundary theory, which is preceded by an abbreviated discussion of pertinent material.

The Wiener compactification $R^{*}$ of $R$ is generated by the Wiener algebra $W(R)$ of bounded, continuous, harmonizable functions on $R$. We also have the class of (bounded) Wiener potentials denoted by $W_{0}(R)$. Refer to Sario-Nakai [10], Chapter IV, for details.

In an analogous vein, associated with the equation $\Delta u=P u$, we have the Wiener $P$-compactification $R_{P}^{*}$, generated by the Wiener $P$-algebra $W_{P}(R)$. See e.g. Constantinescu-Cornea [1], Tanaka [13] for details. Denoting the class of Wiener $P$-potentials by $W_{0 P}(R)$, we obtain (cf. also Maeda [5], Tanaka [12]):

Proposition 10. Under condition $(*), \quad W(R)=W_{P}(R)$, and $W_{0}(R)=W_{0 P}(R)$.

The proof follows from the fact that as we have seen $(*)$ implies $P B(R)$ is canonically isomorphic to $H B(R)$, which implies $h_{P}$, the least harmonic majorant of the elliptic measure is identically one (Lahtinen [3]), which in turn yields $W(R)=W_{P}(R)$ and $W_{0}(R)=W_{0 P}(R)$ (Tanaka [12]).

Since $W_{0}(R)$ and $W_{0 P}(R)$ completely determine $\Delta$ and $\Delta_{P}$ (the Wiener $P$-harmonic boundary) respectively, we have the following useful result.

Corollary. Under condition $(*), \Delta_{P}=\Delta$.

In the sequel we make use of the fact that every continuous $P$-potential on $R$ vanishes on $\Delta_{P}$ (cf. Constantinescu-Cornea [1]).

THEOREM 5. On the unit disk $U$, any density $P$ satisfying condition (*) yields $P B(U) \varsubsetneqq \Lambda^{q}(U), 1<q<\infty$.

Proof. Choose a function $h \in H^{q}(U)-H B(U)$. Let $P$ be a density satisfying $(*)$, so that $\Delta_{P}=\Delta$.

For $u=\lambda h \in \Lambda^{q}(U), h-u$ is a continuous $P$-potential, implying $h-u \mid \Delta_{P}=0$. Hence $h|\Delta=u| \Delta$, and by Proposition 1, since 
$H^{a}(R) \subset H B^{\prime}(R), h$ is unbounded on $\Delta . \quad$ It follows that $u$ is unbounded and $u \in \Lambda^{q}(U)-P B(U)$.

Corollary. $M_{P}^{q}(U) \not \subset H B(U), 1<q<\infty$.

Proof. If $0<u \in \Lambda_{P}^{q}(U)-P B(U)$, then $h^{\prime}=\mu_{P} u \in M_{P}^{q}(U)$ and $h^{\prime} \notin H B(U)$.

Thus, in general, there is no inclusion relation between $H B(R)$ and $M_{P}^{q}(R)$.

In contradistinction to Theorem $3,(*)$ is a sufficient condition for $M^{q}(R)$ to be identical with $H^{q}(R)$.

THEOREM 6. $\lambda_{P}: H^{q}(R) \rightarrow \Lambda_{P}^{q}(R)$ is an isomorphism, i.e. $M^{q}(R)=$ $H^{q}(R)$, whenever condition $(*)$ is valid, $1<q<\infty$.

Proof. Suppose $\lambda_{P} h=0$ for sỏme $h \in H^{a}(R)$. Since $h=h_{1}-h_{2}$, $h_{1} \geqq 0, h_{1} \in H^{q}(R), i=1,2$, we have $\lambda_{P} h_{1}=\lambda_{P} h_{2}$. As $h_{i}-\lambda_{P} h_{1}$ is a $P$-potential, and $\Delta_{P}=\Delta$, it follows that $h_{l}\left|\Delta=\lambda_{P} h_{l}\right| \Delta$. Hence $h_{1} \mid \Delta=$ $h_{2} \mid \Delta$, i.e. $h \mid \Delta=0$. Since $H^{q}(R) \subset H B^{\prime}(R)$ for $1<q<\infty$ (cf. LumerNaim [4]), the maximum principle for $H B^{\prime}(R)$ implies $h \equiv 0$ on $R$, and $\lambda_{P}$ is injective on $H^{a}(R)$.

Our final example concerns the inclusion relation $\Lambda_{P}^{q}(R) \subset P^{q}(R)$.

Proposition 11. There is a Riemann surface $R$ and a density $P$ such that $\Lambda_{P}^{1}(R) \varsubsetneqq P^{1}(R)$.

Proof. Let $R$ be the punctured unit disk $U_{0}$, and for $|z|=r$, $P=P(r)=1 / r^{2}$. Then the function $u=u(r)=1 / r$ satisfies $\Delta u=P u$ on $U_{0}$. Moreover, any positive harmonic function $h$ on $U_{0}$ must be asymptotic to $-a \log r$, at the origin, for some $a \in[0, \infty)$ (cf. eg. Sario-Nakai [10]). Consequently, for any $h \in H\left(U_{0}\right),|u|=1 / r \not \equiv h$ on $U_{0}$, i.e. $u \notin \Lambda_{P}^{1}\left(U_{0}\right)$. However, $u \in P^{1}\left(U_{0}\right)$.

Whether there exists a pair $(R, P)$ such that $\Lambda_{P}^{q}(R) \varsubsetneqq P^{q}(R)$ for $1<q<\infty$, is as yet unresolved.

5. Extensions. In the sequel we assume $P B(R) \neq\{0\}$.

The Wiener algebra $W(R)$ has the following extension. Let $\mathscr{W}(R)$ be the class of continuous harmonizable functions $f$ on $R$ such that there exists a continuous superharmonic function $s_{f}$ with a discrete $\left\{s_{f}=\infty\right\}$ and with $|f| \leqq s_{f}$. Denote by $\mathscr{W}_{P}(R)$ the $P$-harmonic analogue (cf. Constantinescu-Cornea [1]). Clearly $W(R) \subset \mathscr{W}(R)$ and $W_{P}(R) \subset$ $\mathscr{W}_{P}(R)$. Furthermore, define

$$
\mathscr{W}_{0}(R)=\{f \in \mathscr{W}(R): f \mid \Delta=0\},
$$


and

$$
\mathscr{W}_{0 P}(R)=\left\{f \in \mathscr{W}_{P}(R): f \mid \Delta_{P}=0\right\} .
$$

We now establish:

Lemma. If $\mathscr{W}(R)=\mathscr{W}_{P}(R)$, then $\Lambda_{P}^{q}(R)=P^{q}(R), 1 \leqq q<\infty$.

Proof. Suppose not, and let $u \in P^{q}(R)-\Lambda_{P}^{q}(R)$, for some $q, 1 \leqq$ $q<\infty$. Then $|u|^{q} \leqq v$ for some $v \in P(R)$. Since $u$ is also continuous, positive $P$-subharmonic, $|u|^{a} \in \mathscr{W}_{P}(R)$. If moreover, $|u|^{a} \in \mathscr{W}(R)$, then $|u|^{q} \leqq s$ for some superharmonic function $s$ on $R$. Since $|u|^{a}$ is subharmonic, $|u|^{a} \leqq h$ for some $h \in H(R)$, implying $u \in \Lambda_{P}^{q}(R)$, a contradiction. Thus, $|u|^{a} \notin \mathscr{W}(R)$ which yields $\mathscr{W}(R) \neq \mathscr{W}_{P}(R)$, and the result follows.

THEOREM 7. If $\mathscr{W}(R)=\mathscr{W}_{P}(R)$ and $\mathscr{W}_{0}(R)=\mathscr{W}_{0 P}(R)$, then $H^{q}(R)$ is isomorphic to $P^{q}(R), 1<q<\infty$.

Proof. It is easily seen that the hypothesis implies $W(R)=W_{P}(R)$ and $W_{0}(R)=W_{0 P}(R)$, and consequently, $R^{*}=R_{P}^{*}$ and $\Delta=\Delta_{P}$. Under the condition that $\Delta=\Delta_{P}$, Theorem 6 obtains, that is $\lambda_{P}: H^{q}(R) \rightarrow \Lambda_{p}^{q}(R)$ is an isomorphism, for $1<q<\infty$. The theorem now follows from the lemma.

THEOREM 8. If $\mathscr{W}(R)=\mathscr{W}_{P}(R)$ and $\mathscr{W}_{0}(R)=\mathscr{W}_{0 P}(R)$, then $H B^{\prime}(R)$ is isomorphic to $P B^{\prime}(R)$.

Proof. For $u \in P B^{\prime}(R) \subset \mathscr{W}_{P}(R)=\mathscr{W}(R)$, we have the decomposition

$$
u=v+g
$$

where $v \in H B^{\prime}(R)$ and $g \in \mathscr{W}_{0}(R)$ (cf. Sario-Nakai [10]). We define $T: P B^{\prime}(R) \rightarrow H B^{\prime}(R)$ by $T u=v$. Since $\Delta=\Delta_{P}$ and $u|\Delta=T u| \Delta$, it is a simple matter to verify that $T$ is the desired isomorphism.

As we have seen, condition $(*)$ implies $W(R)=W_{P}(R)$, and the question naturally arises whether or not $(*)$ implies $\mathscr{W}(R)=\mathscr{W}_{P}(R)$. That this is not the case is seen as follows.

Let $R_{\alpha}=\{0<|z|<\alpha<1\}$, and $u=u(r)=(\log r)^{2}$, with $r=$ $|z|$. Then $\Delta u=P u$ for $P=P(r)=2 /(r \log r)^{2}$. Since $u$ is continuous, $P$-harmonizable on $R_{\alpha}, u \in \mathscr{W}_{P}\left(R_{\alpha}\right)$. Moreover, if $u$ had a superharmonic majorant on $R_{\alpha}$, then the subharmonicity of $u$ would imply $u$ had a harmonic majorant $h$ on $R_{\alpha}$. As in Proposition 11, $h$ would be 
asymptotic to $-a \log r$ at $z=0$, for $a \in[0, \infty)$, which would violate the fact that $h$ must dominate $u$. We conclude that $u \notin \mathscr{W}\left(R_{\alpha}\right)$.

Finally, a simple calculation shows that

$$
\int_{R_{\alpha}} P d x d y=\frac{-4 \pi}{\log \alpha}<\infty
$$

and hence $\int_{R_{\alpha}} G(z, \zeta) P(\zeta) d \xi d \eta<\infty$. Therefore $(*)$ is valid, but $\mathscr{W}\left(R_{\alpha}\right) \neq \mathscr{W}_{P}\left(R_{\alpha}\right)$.

\section{REFERENCES}

1. C. Constantinescu and A. Cornea, Compactification of harmonic spaces, Nagoya Math. J., 25 (1965), 1-57.

2. M. Heins, Hardy Classes on Riemann Surfaces, Forschungsinstitut für Mathematikseries Berlin-Heidelberg-New York: Springer 1969.

3. A. Lahtinen, On the solutions of $\Delta u=P u$ for acceptable densities on open Riemann surfaces, Ann. Acad. Sci: Fenn. A. I., 515 (1972).

4. L. Lumer-Naim, $H^{p}$ spaces of harmonic functions, Ann. Inst. Fourier, 17 (1967), 425-469.

5. F. Y. Maeda, Comparison of the classes of Wiener functions, J. Sci. Hiroshima Univ., Ser A. I., 33 (1969), 231-235.

6. M. Nakai, The equation $\Delta u=P u$ on the unit disk with almost rotation free $P \geqq 0$, J. Differential Equations, 11 (1972), 307-320.

7. — Order comparisons on canonical isomorphisms, Nagoya Math. J., 50 (1973), 67-87.

8. M. Parreau, Sur les moyennes des fonctions harmoniques et analytiques et la classification des surfaces de Riemann, Ann. Inst. Fourier, 3 (1951), 103-197.

9. H. L. Royden, The equation $\Delta u=P u$ and the classification of open Riemann surfaces, Ann. Acad. Sci. Fenn., 271 (1959).

10. L. Sario and M. Nakai, Classification Theory of Riemann Surfaces, Berlin-Heidelberg-New York: Springer 1970.

11. J. L. Schiff, $H^{p}$-spaces of harmonic functions and the Wiener compactification, Math. Z., 132 (1973), 135-140.

12. H. Tanaka, A remark on Wiener functions for the equation $\Delta u=p u$, Sci. Rep. Saitama Univ., Ser. A VII, No. 3 (1973), 15-24.

13. - On Wiener compactification of a Riemann surface associated with the equation $\Delta u=P u$, Proc. Japan Acad., 45 (1969), 675-679.

Received September 29, 1975.

UNIVERSITY OF AUCKLAND

University of CAlifornia, Los Angeles

AND

Western Washington State College 


\section{PACIFIC JOURNAL OF MATHEMATICS}

\section{EDITORS}

RICHARD ARENS (Managing Editor)

University of California

Los Angeles, California 90024

\section{J. Dugundu}

Department of Mathematics University of Southern California Los Angeles, California 90007

D. Gilbarg and J. Milgram Stanford University Stanford, California 94305

\section{ASSOCIATE EDITORS}
E. F. BECKENBACH
B. H. NeumanN
F. WOLF
K. YoSHIDA

\section{SUPPORTING INSTITUTIONS}

UNIVERSITY OF BRITISH COLUMBIA CALIFORNIA INSTITUTE OF TECHNOLOGY

UNIVERSITY OF CALIFORNIA

MONTANA STATE UNIVERSITY

UNIVERSITY OF NEVADA

NEW MEXICO STATE UNIVERSITY

OREGON STATE UNIVERSITY

UNIVERSITY OF OREGON

OSAKA UNIVERSITY

\author{
UNIVERSITY OF SOUTHERN CALIFORNIA \\ STANFORD UNIVERSITY \\ UNIVERSITY OF HAWAII \\ UNIVERSITY OF TOKYO \\ UNIVERSITY OF UTAH \\ WASHINGTON STATE UNIVERSITY \\ UNIVERSITY OF WASHINGTON \\ AMERICAN MATHEMATICAL SOCIETY
}

The Supporting Institutions listed above contribute to the cost of publication of this Journal, but they are not owners or publishers and have no responsibility for its contents or policies.

Mathematical papers intended for publication in the Pacific Journal of Mathematics should be in typed form or offset-reproduced (not dittoed), double spaced with large margins. Underline Greek letters in red, German in green, and script in blue. The first paragraph or two must be capable of being used separately as a synopsis of the entire paper. Items of the bibliography should not be cited there unless absolutely necessary, in which case they must be identified by author and Journal, rather than by item number. Manuscripts, in duplicate, may be sent to any one of the four editors. Please classify according to the scheme of Math. Reviews, Index to Vol. 39. All other communications should be addressed to the managing editor, or Elaine Barth, University of California, Los Angeles, California, 90024.

100 reprints are provided free for each article, only if page charges have been substantially paid. Additional copies may be obtained at cost in multiples of 50 .

The Pacific Journal of Mathematics is issued monthly as of January 1966. Regular subscription rate: $\$ 72.00$ a year (6 Vols., 12 issues). Special rate: $\$ 36.00$ a year to individual members of supporting institutions.

Subscriptions, orders for back numbers, and changes of address should be sent to Pacific Journal of Mathematics, 103 Highland Boulevard, Berkeley, California, 94708.

PUBLISHED BY PACIFIC JOURNAL OF MATHEMATICS, A NON-PROFIT CORPORATION

Printed at Jerusalem Academic Press, POB 2390, Jerusalem, Israel.

$$
\begin{gathered}
\text { Copyright } 1976 \text { Pacific Journal of Mathematics } \\
\text { All Rights Reserved }
\end{gathered}
$$




\section{Pacific Journal of Mathematics}

Vol. 62, No. 2

February, 1976

Allan Russell Adler and Catarina Isabel Kiefe, Pseudofinite fields, procyclic

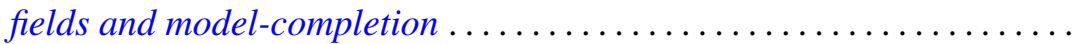

Christopher Allday, The stratification of compact connected Lie group

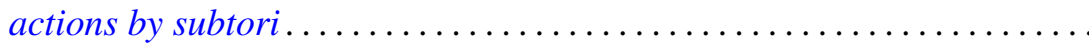

Martin Bartelt, Commutants of multipliers and translation operators .......

Herbert Stanley Bear, Jr., Ordered Gleason parts ..................

James Robert Boone, On irreducible spaces. II .....................

James Robert Boone, On the cardinality relationships between discrete

collections and open covers ............................

L. S. Dube, On finite Hankel transformation of generalized functions .......

Michael Freedman, Uniqueness theorems for taut submanifolds . . . . . . . . .

Shmuel Friedland and Raphael Loewy, Subspaces of symmetric matrices

containing matrices with a multiple first eigenvalue .............

Theodore William Gamelin, Uniform algebras spanned by Hartogs

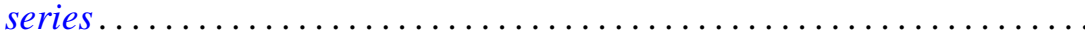

James Guyker, On partial isometries with no isometric part ............

Shigeru Hasegawa and Ryōtarō Satō, A general ratio ergodic theorem for

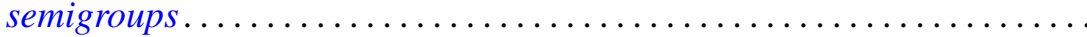

Nigel Kalton and G. V. Wood, Homomorphisms of group algebras with norm less than $\sqrt{2}$.

Thomas Laffey, On the structure of algebraic algebras...

Will Y. K. Lee, On a correctness class of the Bessel type differential operator $S_{\mu}$

Robert D. Little, Complex vector fields and divisible Chern classes ....

Kenneth Louden, Maximal quotient rings of ring extensions . .

Dieter Lutz, Scalar spectral operators, ordered $l^{\rho}$-direct sums, and the

counterexample of Kakutani-McCarthy . .

Ralph Tyrrell Rockafellar and Roger Jean-Baptiste Robert Wets, Stochastic

convex programming: singular multipliers and extended duality

singular multipliers and duality.

Edward Barry Saff and Richard Steven Varga, Geometric overconvergence of rational functions in unbounded domains ..........

Joel Linn Schiff, Isomorphisms between harmonic and P-harmonic Hardy

spaces on Riemann surfaces.

Virinda Mohan Sehgal and S. P. Singh, On a fixed point theorem of

Krasnoselskii for locally convex spaces.

Lewis Shilane, Filtered spaces admitting spectral sequence operations

Michel Smith, Generating large indecomposable continua . 